\title{
Solar atmosphere neutrino oscillations
}

\author{
G. L. Fogli ${ }^{\mathrm{a}}$, E. Lisi ${ }^{\mathrm{a}}$, A. Mirizzi ${ }^{\mathrm{a}}$, D. Montanino ${ }^{\mathrm{b}}$, and P. D. Serpico ${ }^{\mathrm{c}}$ \\ ${ }^{a}$ Dipartimento di Fisica and Sezione INFN, Via Amendola 173, 70126 Bari, Italy

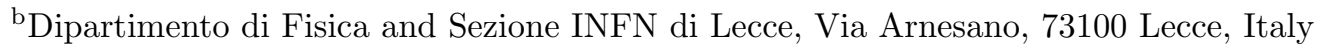 \\ ${ }^{\mathrm{c}}$ Particle Astrophysics Center, Fermi National Accelerator Laboratory, Batavia, IL 60510-0500 USA
}

The Sun is a source of high energy neutrinos $(E>10 \mathrm{GeV})$ produced by cosmic ray interactions in the solar atmosphere. We study the impact of three-flavor oscillations on the solar atmosphere neutrino fluxes observable at Earth. We find that peculiar matter oscillation effects in the Sun do exist, but are significantly suppressed by averaging over the production region and over the neutrino and antineutrino components. In particular, the relation between the neutrino fluxes at the Sun and at the Earth can be approximately expressed in terms of phase-averaged "vacuum" oscillations, dominated by a single mixing parameter (the angle $\theta_{23}$ ).

FERMILAB-CONF-07-022-A

1. Introduction - The Sun is a source of high energy neutrinos $(E>10 \mathrm{GeV})$ generated when cosmic rays interact in the solar atmosphere [1-4], producing secondary mesons which subsequently decay into $\nu_{e}, \bar{\nu}_{e}, \nu_{\mu}$, and $\bar{\nu}_{\mu}$, collectively referred to as "solar atmosphere neutrinos" (SA $\nu)$ hereafter. At the Earth, within the solid angle subtended by the Sun, the $\mathrm{SA} \nu$ flux significantly exceeds the familiar "atmospheric neutrino" flux generated by cosmic rays interacting with the Earth atmosphere: the density at the first interaction point is lower in the solar atmosphere than in the terrestrial one, therefore a larger fraction of mesons decay into neutrinos instead of being absorbed.

The characterization of the $\mathrm{SA} \nu$ signal is an important goal, both in itself and because it might represent a "background" in searches for signatures of possible weakly interacting massive particle (WIMP) annihilation in the Sun. Here we characterize the impact of flavor transitions (in vacuum and in matter) on $\mathrm{SA} \nu$. In Sec. 2 we describe the input $\mathrm{SA} \nu$ fluxes at the Sun. In Sec. 3 we study $\mathrm{SA} \nu$ oscillations in vacuum and in matter within the standard $3 \nu$ framework. In Sec. 4 we evaluate the impact of flavor oscillations on

\footnotetext{
*Speaker. E-mail: alessandro.mirizzi@ba.infn.it
}

the observable events rates in a $\mathrm{km}^{3}$ neutrino telescope. We conclude in Sec. 5. This talk in based on the result of [5] to which we address the interested reader for further details.

2. Solar atmosphere neutrino fluxes The most recent calculation of the $\mathrm{SA} \nu$ flux has been performed in [3] by using Monte Carlo codes for high-energy particle interactions. From pion production dominance, at the source the $\mathrm{SA} \nu$ flavor ratio is expected to be $\phi_{e}: \phi_{\mu}: \phi_{\tau} \simeq 1$ : $2: 0$, with approximately equal fluxes of $\nu$ and $\bar{\nu}$. The simulations in [3] find a power-law decrease $\sim E^{-3}$ for the $\mathrm{SA} \nu$ energy spectrum at $E>100 \mathrm{GeV}$. At lower energies, heliomagnetic effects are expected to harden the spectrum (as $\sim E^{-2}$ ) [4]. After production, SA $\nu$ 's are affected by the solar medium through both absorption on nucleons and oscillations in matter. In the energy range of interest for our work, absorption can be accounted for by an overall attenuation function multiplying the original $\nu$ fluxes [3], while oscillation effects can be applied afterwards.

3. Solar atmosphere neutrino oscillations - Solar atmosphere neutrinos are affected by flavor oscillations (both in matter and in vacuum) during their propagation. Within the standard $3 \nu$ framework, the oscillation parameters include two squared mass differences $\left(\delta m^{2}, \pm \Delta m^{2}\right)$ and three 

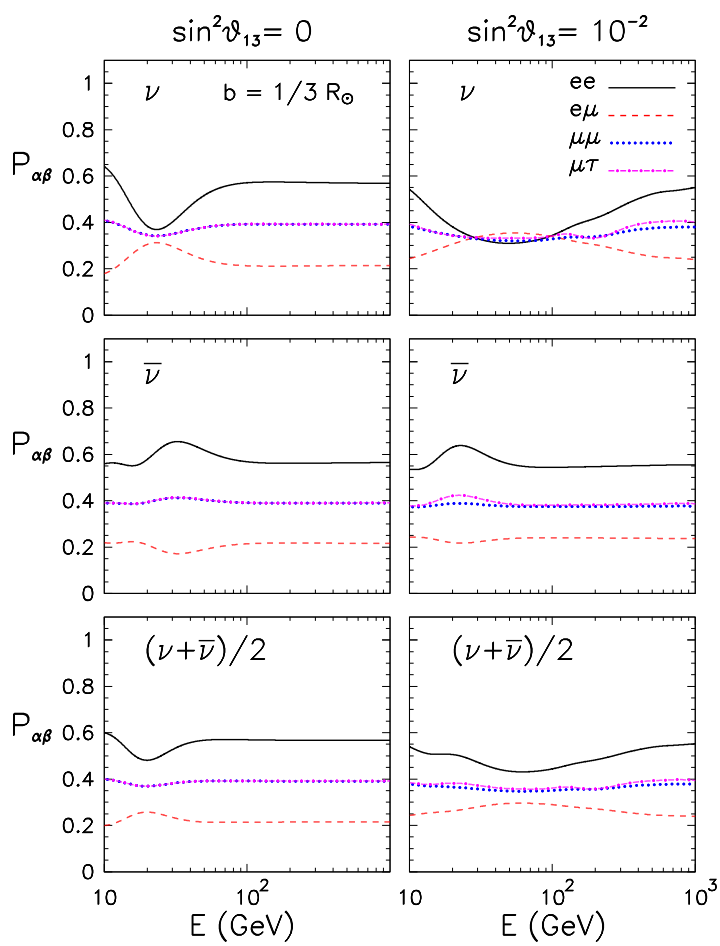

Figure 1. Oscillation probabilities for solar atmosphere neutrinos as functions of energy, for $\sin ^{2} \theta_{13}=0$ (left panels) and $\sin ^{2} \theta_{13}=10^{-2}$ (right panels) at fixed impact parameter $b=1 / 3 R_{\odot}$.

mixing angles $\left(\theta_{12}, \theta_{23}, \theta_{13}\right)$, whose ordered rotations form a unitary mixing matrix $U$. We take their best-fit values and $2 \sigma$ ranges from [6]. In the following, we assume the normal mass hierarchy $\left(+\Delta m^{2}\right)$. In matter, the $\nu$ oscillation dynamics also depends on the $\nu_{e}-\nu_{\mu, \tau}$ interaction energy difference $\pm V$ (where $+/$ - for $\nu / \bar{\nu}$ ), which is proportional to the electron density along the path. Potentially large matter effects are expected when one of the "resonance conditions" is realized, roughly when $V \sim \delta m^{2} / 2 E$ or $V \sim \Delta m^{2} / 2 E$.

To evaluate the $\mathrm{SA} \nu$ oscillation probabilities $P_{\alpha \beta}$, we evolve numerically the $\nu$ and $\bar{\nu}$ flavor evolution equations for many different values of the impact parameter $b$ of the neutrino trajectory $\left(0 \leq b \leq R_{\odot}\right)$. From the unitarity property of the probabilities, one can express all the $P_{\alpha \beta}$ 's in terms of only four independent quantities. In the following, we choose $P_{e e}, P_{e \mu}, P_{\mu \mu}$ and $P_{\mu \tau}$ as independent set.

In Figure 1 we plot these probabilities as functions of neutrino energy $E$ after gaussian energy resolution smearing (with $\Delta E / E=10 \%$ for definiteness), for a representative impact parameter $b=1 / 3 R_{\odot}$, and for both $\sin ^{2} \theta_{13}=0$ (left panels) and $\sin ^{2} \theta_{13}=10^{-2}$ (right panels), assuming maximal 2-3 mixing and normal mass hierarchy in both cases. The upper and middle panels refer to neutrinos and antineutrinos, respectively. Since solar atmosphere $\nu$ 's and $\bar{\nu}$ 's have approximately equal fluxes, and are basically indistinguishable in neutrino telescopes, we find it useful to plot the arithmetic average of the $\nu$ and $\bar{\nu}$ oscillation probabilities in the lower panels of Fig. 1. For $\sin ^{2} \theta_{13}=0$ (left panels of Fig. 1), the energy-flat vacuum limit $(V=0)$ is reached for $E>50 \mathrm{GeV}$. Matter effects emerge around $E \sim 20 \mathrm{GeV}$, and are more pronounced in the neutrino channel (where the $\delta m^{2}$-induced resonance can be realized). For $\sin ^{2} \theta_{13}=10^{-2}$ (right panels of Fig. 1), additional three-flavor mixing effects, due to a $\Delta m^{2}$-induced resonance, are seen to emerge in the neutrino channel around $E \sim$ $100 \mathrm{GeV}$, with broad features entangled with the previous, lower resonance range. As compared with the left panels, the vacuum limit is reached at higher energies, but with almost the same limiting values for $P_{\alpha \beta}$. The two lowest panels in Fig. 1 show that matter effects, averaged over $\nu$ and $\bar{\nu}$ components, produce only mild variations with respect to the energy-flat vacuum limit, independently of the value of $\sin ^{2} \theta_{13}$. Integration over the impact parameter $b$ (see Fig. 5 in [5]) strengthens these conclusions, since trajectories with relatively high impact parameter $b$ (and thus with short path in the Sun and small matter effects) have large geometrical weight. The energy dependence of all probabilities is rather flat and roughly insensitive to $\sin ^{2} \theta_{13}$, with only minor deviations from the vacuum limit governed by the parameter $\sin ^{2} \theta_{23}$. For the specific case of 2-3 maximal mixing $\left(\sin ^{2} \theta_{23}=1 / 2\right)$, one would recover the well-known result for the phaseaveraged, vacuum neutrino flavor ratio at detection $\phi_{e}^{D}: \phi_{\mu}^{D}: \phi_{\tau}^{D} \simeq 1: 1: 1$. The current error 
on $\sin ^{2} \theta_{23}$ represents the main uncertainty associated to oscillation effects in calculating $\mathrm{SA} \nu$ fluxes. To give an example, the $\pm 2 \sigma$ spread on $\sin ^{2} \theta_{23}$ produces a $\pm 15 \%$ variation on the flavor ratio $\phi_{e}^{D} / \phi_{\mu}^{D}$.

4. Event rates at Earth - Solar atmosphere neutrinos may be observable in largevolume detectors. Figure 2 shows the results of our calculation for the muon and tau event rates (summing over $\nu$ and $\bar{\nu}$ ) in a $\mathrm{km}^{3}$ detector like IceCube, as functions of the threshold energy $E_{\mathrm{th}}$, including oscillations in matter. A low energy threshold $E_{\text {th }} \lesssim 100 \mathrm{GeV}$ is crucial to get a handful of events per year in km-sized detectors. However, even a few $\mathrm{SA} \nu$-induced muon events per year at $E \gtrsim 100 \mathrm{GeV}$ (vs. a prediction twice as large in the absence of $\theta_{23}$-driven oscillations) might allow an independent check in a decade of the large 2-3 mixing established by terrestrial atmospheric neutrinos. This is particularly viable since the theoretically calculated normalization of the $\mathrm{SA} \nu$ flux can be checked indirectly by the GLAST satellite via the measurements of $\gamma$ 's coming from $\pi_{0}$ decays, produced in the very interactions generating the $\mathrm{SA} \nu$ flux [1].

5. Conclusions - We have recently studied solar atmosphere neutrino oscillations in detail, in the light of the most recent determinations of the neutrino mass mixing parameters, and paying particular attention to matter effects (generally neglected a priori in this context) [5]. We found that, in the energy range $E \in[10,1000] \mathrm{GeV}$ relevant for detection, $\mathrm{SA} \nu$ oscillations in matter have in principle a rich phenomenology. Unfortunately, these effects are smeared out to a large extent by summing over the neutrino and antineutrino channel, and by integrating over the solar disk angle (as well as over energy). In practice, oscillations are dominated by the "vacuum" effect of the $\theta_{23}$ mixing angle. Therefore, even if matter effects can and do occur, the original flavor ratio $\phi_{e}: \phi_{\mu}: \phi_{\tau} \simeq 1: 2: 0$ at the source approximately yields the well-known ratio $\phi_{e}^{D}: \phi_{\mu}^{D}: \phi_{\tau}^{D} \simeq 1: 1: 1$ at the detector (for maximal 2-3 mixing), just as in the case of phase-averaged vacuum oscillations.

Even if only a handful of SA $\nu$ events per year may be detectable in a $\mathrm{km}^{3}$ telescope, their the-

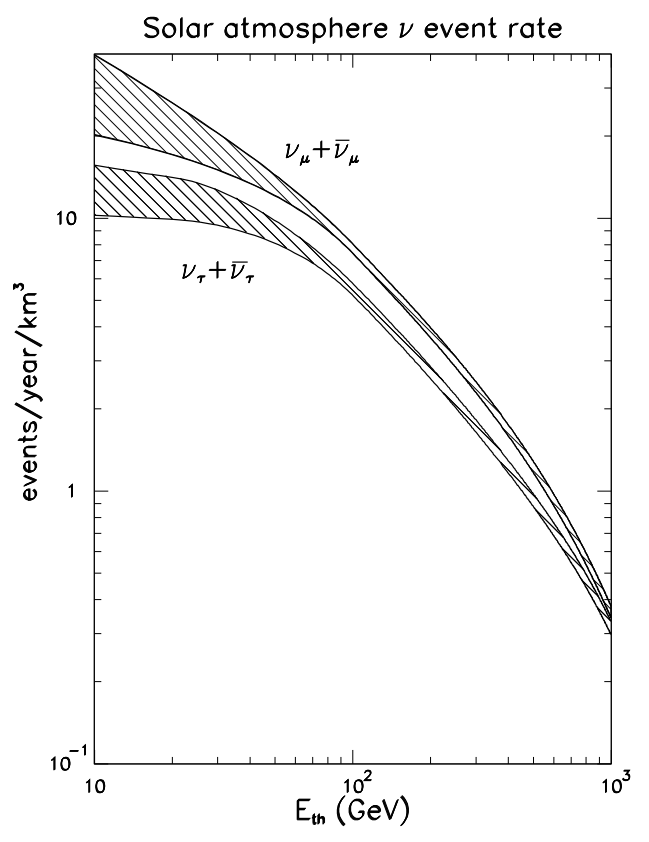

Figure 2. $\nu_{\mu}$ and $\nu_{\tau}$ event rate in a $\mathrm{km}^{3}$ detector vs. the detector threshold energy $E_{\mathrm{th}}$. The $\nu_{e}$ event rate (not shown) is very similar to that of muon events. The width of the bands includes the uncertainties associated to $\sin ^{2} \theta_{23}$ and to the initial fluxes [5].

oretical fluxes are relatively under control, and thus a measurement might provide a high-energy, astrophysical probe of neutrino flavor mixing.

Acknowledgments - PS acknowledges support by the US Department of Energy and by NASA grant NAG5-10842.

\section{REFERENCES}

1. D. Seckel, T. Stanev, and T. K. Gaisser, Astrophys. J. 382, 652 (1991).

2. I. V. Moskalenko and S. Karakula, J. Phys. G 19, 1399 (1993).

3. G. Ingelman and M. Thunman, Phys. Rev. D 54, 4385 (1996).

4. C. Hettlage, K. Mannheim and J. G. Learned, Astropart. Phys. 13, 45 (2000).

5. G. L. Fogli et al., Phys. Rev. D 74, 093004 (2006).

6. G. L. Fogli et al., hep-ph/0608060. 